\title{
Non-Randomized Trial
}

\section{e Radiofrequency Thermocoagulation Through Foramen Rotundum Versus Foramen Ovale for the Treatment of V2 Trigeminal Neuralgia}

\author{
Tong-qing Xue, PhD', Qian-xi Zhang, MD², Hu Bian, BD³, Peng-cheng Zhou, BD', \\ Cheng Liu, BD'1, Shang-fu Niu, MD', Zhi-bing Wang, MD', Wen-jie Shi, BD', \\ and Cong-yang Yan, BD ${ }^{5}$
}

From: 'Department of Pain and Intervention Management, Huaian Hospital of Huaian City, Huaian,

Jiangsu, China; ${ }^{2}$ Department

of Pain Management, Huaian Second People's Hospital, Huaian,

Jiangsu, China; ${ }^{3}$ Department of

Pain and Intervention, Huaiyin Hospital of Huaian City, Huaian,

Jiangsu, China; ${ }^{4}$ Department of Psychiatry, Huaian No. 3 People's Hospital, Huaian, Jiangsu, China;

${ }^{5}$ Department of Neurology, Lianshui People's Hospital, Huaian, Jiangsu, China

Address Correspondence: Cong-yang Yan, BD Department of Neurology Lianshui People's Hospital No. 6 Hongri Rd. Lianshui County, Huaian Jiangsu 223400, China E-mail:

congyang_med@126.com

Disclaimer: Tong-qing Xue, Qian-xi Zhang, and Hu Bian contributed equally to this study. There was no external funding in the preparation of this manuscript.

Conflict of interest: Each author certifies that he or she, or a member of his or her immediate family, has no commercial association (i.e., consultancies, stock ownership, equity interest, patent/licensing arrangements, etc.) that might pose a conflict of interest in connection with the submitted manuscript.

Manuscript received: 11-27-2018 Revised manuscript received: 02-27-2019

Accepted for publication: 04-08-2019

Free full manuscript: www.painphysicianjournal.com
Background: Percutaneous radiofrequency thermocoagulation through the foramen rotundum (FR) is a new approach for the treatment of V2 trigeminal neuralgia (TN).

Objectives: This study aimed to compare the efficacy and safety of the FR approach with that of the foramen ovale (FO) approach.

Study Design: Nonrandomized controlled clinical trial.

Setting: The study was conducted at Huaian Hospital of Huaian City, Huaian, China.

Methods: From July 2014 to December 2016, 80 consecutive patients with V2 TN were prospectively assigned into the FO group $(n=40)$ or the FR group $(n=40)$. All radiofrequency thermocoagulation procedures were performed under the guidance of digital subtraction angiography (DSA). Patients in the FO group were treated with Gasserian ganglion ablation through the Hartel approach. Patients in the FR group received ablation of the maxillary nerve at the internal opening of the FR. Facial pain was evaluated using the Visual Analog Scale preoperatively and postoperatively at 1 week, 6 months, and 1 year.

Results: All surgical procedures were successfully completed using DSA guidance. The FR group had no facial pain at postoperative 1 week, 6 months, and 1 year. The facial fain was not relieved in 4 patients of the FO group. They were treated with radiofrequency thermocoagulation of the maxillary nerve through the FR and maintained painless at postoperative 1 week, 6 months, and 1 year. At postoperative 1 year, another 3 patients relapsed in the FO group. The incidences of facial numbness and swelling did not differ significantly between the 2 groups (all $P>0.05$ ). There was no postoperative corneal involvement or masticatory weakness in the FR group. However, corneal involvement and masticatory weakness occurred postoperatively in 22 (55\%) patients and 31 (77.5\%) patients in the FO group. The FR group had significantly shorter operation time than the FO group $(19.3 \pm 5.9$ vs. $32.7 \pm 8.7$ minutes; $P<0.05)$.

Limitations: We were unable to avoid the $\mathrm{V} 1$ and $\mathrm{V} 3$ branches, despite multiple adjustments of the needed position in 35 of the 40 patients in this group.

Conclusions: For the treatment of V2 TN, thermocoagulation of the maxillary nerve through the FR had better efficacy and fewer complications in comparison with the Gasserian ganglion ablation through the FO.

Key words: Neuralgia, pain, radiology, facial pain

Pain Physician 2019: 22:E609-E614 
$\mathrm{T}$ he maxillary branch (V2) of the trigeminal nerve is most frequently involved in primary trigeminal neuralgia (TN) (1-3). Gasserian ganglion ablation with percutaneous radiofrequency thermocoagulation through the foramen ovale (FO) is the most commonly used treatment for TN (4-6). This procedure is effective for TN caused by any of the 3 branches. However, ablation of the Gasserian ganglion may cause masticatory weakness and corneal involvement. In addition, Gasserian ganglion ablation through the FO is associated with unsatisfactory efficacy or relapse at 6 months in some patients.

During the procedure of Gasserian ganglion ablation, electrical stimulation and multiple adjustment of the radiofrequency needle position are required to elicit a paresthesia area overlapping the pain area. It is quite difficult to elicit the V2 paresthesia, which may cause treatment failure $(7,8)$. Multiple adjustment of the needle position also increases the risks for intracranial neurovascular injury.

Percutaneous radiofrequency thermocoagulation through the foramen rotundum (FR) has been proposed as a new approach for the treatment of V2 TN $(8,9)$. However, it is suggested that shallow reaching in the FR may be associated with incomplete pain relief. We have developed a novel technique to allow reaching the internal opening of the FR through the infrazygomatic approach. Here, we report our prospective study comparing the FR approach versus the FO approach in the radiofrequency thermocoagulation treatment of V2 TN.

\section{Methods}

\section{Patients}

From July 2014 to December 2016, 80 consecutive patients with V2 TN were treated at our hospital. All patients were confirmed with diagnostic block. The patients were assigned into the FO group $(n=40)$ or the FR group $(n=40)$. All the patients had failed previous medication treatment or surgery or had relapsed. Informed consent was obtained from each patient. Our study was approved by the ethics review board of Huaian Hospital of Huaian City, Huaian, China.

\section{Procedure of the FR Approach}

The patients were put into the supine position on the digital subtraction angiography (DSA) table with the head secured with tape. The needle entry point was inferior to the anterior end of the zygomatic bone. The skin at the entry point was anesthetized with $5 \mathrm{~mL} 1 \%$ lidocaine. A 21-gauge, $10-\mathrm{cm}$ radiofrequency needle with a $10-\mathrm{mm}$ naked needle tip was used. The naked part of the needle tip was bent to form a 40-degree angle. To visualize the FR, the C-arm of the DSA machine was adjusted to get a posteroanterior x-ray projection. The FR was the 3-mm lucent spot in the maxillary sinus shadow that was medially superior to the petrous part of the temporal bone (Fig. 1A). Then the C-arm was adjusted to get a normal position on fluoroscopy. After entering the skin, the radiofrequency needle direction was adjusted, and the needle was advanced toward the FR with the tip immediately pressing the maxilla. The needle tip in the lucent FR shadow indicated that it was inside the pterygopalatine fossa and was positioned anterior to the FR (Fig. 1B). The C-arm was adjusted to get a posteroanterior $x$-ray projection using the Waters projection. A round lucent shadow was seen lateral to the sphenoid sinus, usually superior to the petrous part of the temporal bone. By adjusting the fluoroscopy monitoring angle, the normal position and the lateral position were alternatively viewed. Meanwhile, the needle was rotated to adjust its direction to be posterior toward the FR and parallel to the FR canal. Then, the needle tip was slowly advanced into the FR (Fig. 1C). Then, in the standard lateral view, the bases of the bilateral middle cranial fossa were overlapped. The needle tip was slowly advanced to reach the cortex line of the middle cranial fossa (Fig. 1D). The patient was warned about significant pain caused by the needle tip touching the maxillary nerve, which was managed with $0.1 \mathrm{~mL} 2 \%$ lidocaine through the needle. Threedimensional images were reconstructed using DSA and computed tomography to confirm the route of the needle and the position of the needle tip in the internal opening of the FR (Fig. 2).

The core of the radiofrequency needle was withdrawn, and the electrode was inserted (ET-20S, Smith \& Nephew, Andover, MA). Sensory electrical stimulation at $50 \mathrm{~Hz}$ was started from $0 \mathrm{~V}$ to $1.0 \mathrm{~V}$, then motor electrical stimulation at $2 \mathrm{~Hz}$ was started from $0 \mathrm{~V}$ to $1.0 \mathrm{~V}$. If there was no paresthesia of the ophthalmic nerve and the mandibular nerve when the electrical stimulation reached $1.0 \mathrm{~V}$, it indicated that thermocoagulation may not injure these 2 nerves. Then, $0.3 \mathrm{~mL} 2 \%$ lidocaine was slowly injected through the needle. The patient would feel pain relief and numbness in the maxillary nerve dermatome. The radiofrequency electrode was inserted and thermocoagulation was conducted at $90^{\circ} \mathrm{C}$ for 240 seconds. 


\section{Radiofrequency Thermocoagulation Treatment of V2 Trigeminal Neuralgia}
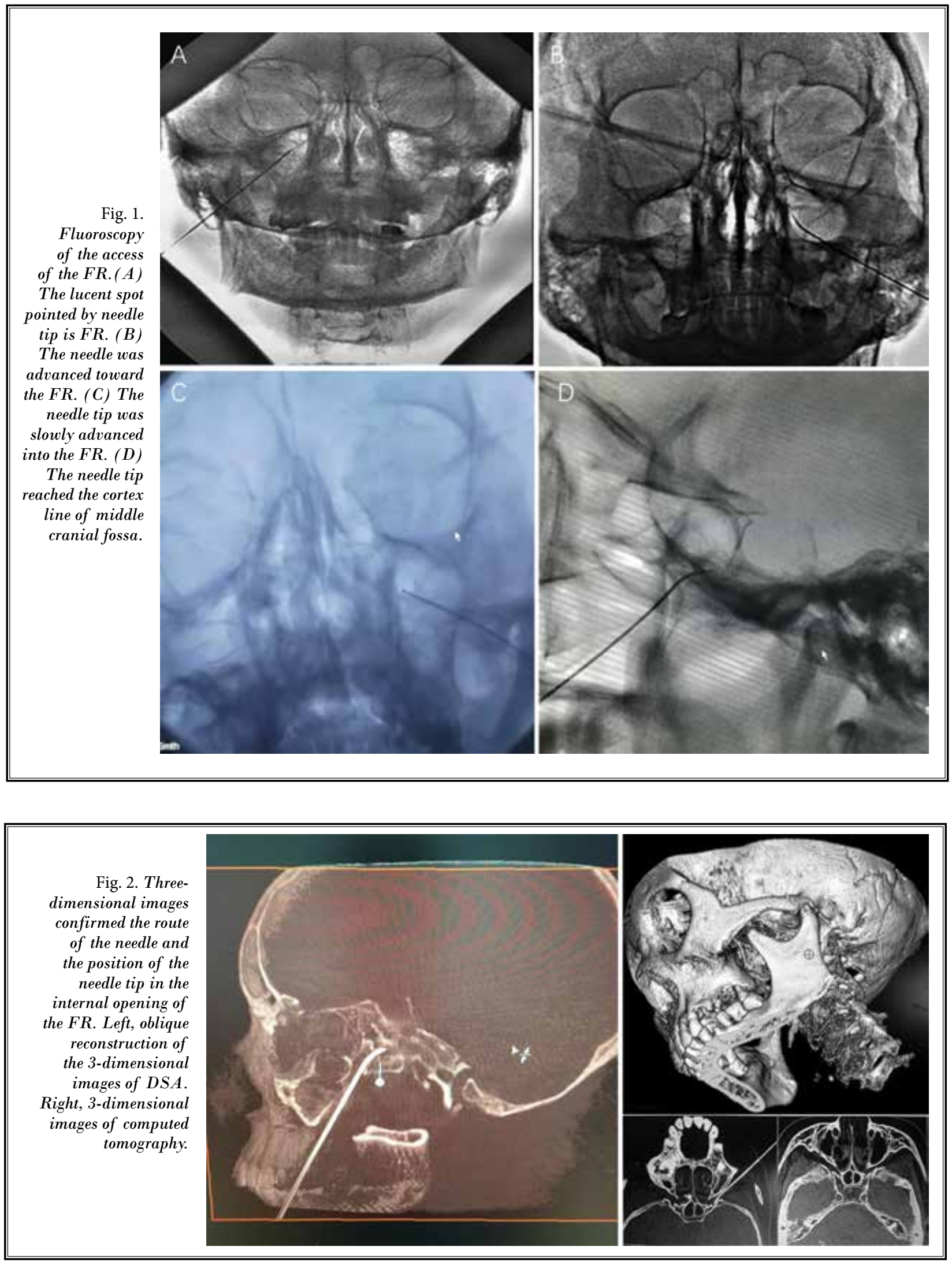


\section{Procedure of the FO Approach}

Patients in the FO group were treated with Gasserian ganglion thermocoagulation through the Hartel approach. A 21-gauge, 10-cm radiofrequency needle with a $10-\mathrm{mm}$ naked section was inserted into the skin $2 \mathrm{~cm}$ lateral to the mouth corner. The needle tip was toward the ipsilateral pupil in the front view and toward the temporomandibular joint in the lateral view. Under the guidance of DSA fluoroscopy, the needle tip was advanced into the FO. The position of the needle was confirmed using 3-dimensional images. Thermocoagulation was conducted at $75^{\circ} \mathrm{C}$ for 240 seconds.

\section{Patient Evaluation and Follow-Up}

Facial pain was assessed using the Visual Analog Scale (VAS) preoperatively and postoperatively during follow-up. Patients were followed up at 1 week, 6 months, and 1 year. Postoperative complications were recorded during the 1-year follow-up. Postoperative ipsilateral facial pain was treated with percutaneous block of the maxillary nerve through the pterygopalatine fossa. If the pain disappeared, it was considered a relapse; otherwise, it was considered pathology of the other branches.

\section{Statistical Analyses}

Continuous data were presented as means and standard deviations and were compared using the Student t test. Categorical data were presented as frequencies and percentages and were compared using the chi-squared test. All statistical analyses were performed using the SPSS Version 19.0 software (IBM Corporation, Armonk, NY). P value $<0.05$ was considered statistically significant.

\section{Results}

There was no significant difference in gender, age, and preoperative VAS scores between the FR group and the FO group (all $P$

Table 1. Patient general information and treatment outcomes.

\begin{tabular}{|l|c|c|c||}
\hline & $\begin{array}{c}\text { FR group } \\
(\mathbf{n}=\mathbf{4 0})\end{array}$ & $\begin{array}{c}\text { Fo group } \\
(\mathbf{n = 4 0 )}\end{array}$ & P Value \\
\hline Age (years) & $64.95 \pm 12.50$ & $64.28 \pm 11.89$ & $>0.05$ \\
\hline Male & $16(40 \%)$ & $24(60 \%)$ & $>0.05$ \\
\hline Operation time (min) & $19.3 \pm 5.9$ & $32.7 \pm 8.7$ & $<0.05$ \\
\hline Preoperative VAS scores & $7.18 \pm 1.64$ & $7.15 \pm 1.53$ & $>0.05$ \\
\hline No facial pain at postoperative 1 week & $40(100 \%)$ & $36(90 \%)$ & $<0.05$ \\
\hline No facial pain at postoperative 6 months & $40(100 \%)$ & $36(90 \%)$ & $<0.05$ \\
\hline No facial pain at postoperative 1 year & $40(100 \%)$ & $33(82.5 \%)$ & $<0.05$ \\
\hline Facial numbness & $40(100 \%)$ & $40(100 \%)$ & $>0.05$ \\
\hline Facial swelling & $14(35 \%)$ & $15(35 \%)$ & $>0.05$ \\
\hline Corneal involvement & 0 & $22(55 \%)$ & $<0.05$ \\
\hline Masticatory weakness & 0 & $31(77.5 \%)$ & $<0.05$ \\
\hline
\end{tabular}

$>0.05$; Table 1). All surgical procedures were successful in the 80 patients.

All 40 patients in the FR group had no facial pain at postoperative 1 week, 6 months, and 1 year. The facial fain was not relieved in 4 patients of the FO group. They were treated with radiofrequency thermocoagulation of the maxillary nerve through the $F R$, and maintained painless at postoperative 1 week, 6 months, and 1 year. At postoperative 1 year, another 3 patients relapsed in the $\mathrm{FO}$ group.

All the 80 patients had numbness in the maxillary nerve dermatome. Facial swelling occurred in 14 patients in the FR group and 15 patients in the FO group $(P>0.05)$, which resolved spontaneously within postoperative 1 week. There was no postoperative corneal involvement or masticatory weakness in the FR group. However, corneal involvement and masticatory weakness occurred postoperatively in $22(55 \%)$ patients and 31 (77.5\%) patients in the FO group. The FR group also had significantly shorter operation time than the FO group $(P<0.05)$.

\section{Discussion}

In our study, the radiofrequency needle tip reached the internal opening of the FR. This ensured more complete ablation of the maxillary nerve than the FO approach. The FR is quite narrow, and therefore the needle inside the FR was very close to the nerve fibers. However, we had treated another 5 patients with V2 TN with radiofrequency thermocoagulation at the external opening of the FR. Four of the 5 patients showed inadequate pain relief. We speculate that ablation at the external opening of FR may result in poor treatment outcomes because of the longer distance from the Gasserian ganglion than that at the internal opening.

Nerve fibers that conduct pain sensation are more sensitive to thermocoagulation than other sensory fibers and motor fibers. Therefore, the pain nerve fibers are first ablated at high temperature. The temperature of thermoco- 
agulation for the treatment of TN is between $70^{\circ} \mathrm{C}$ and $75^{\circ} \mathrm{C}(10,11)$. It has been shown that this temperature range can effectively ablate the pain nerve fibers and spare the motor fibers, thus blocking the conduction of pain sensation (12-14). The Gasserian ganglion contains all the sensory neurons of the 3 branches and the motor nerve fibers innervating the masseter muscles. In our study, the FO group was treated with Gasserian ganglion radiofrequency ablation. However, we were unable to avoid the V1 and V3 branches, despite multiple adjustments of the needed position in 35 of the 40 patients in this group. Therefore, the $75^{\circ} \mathrm{C}$ temperature was selected for the radiofrequency ablation considering the treatment efficacy and possible complications.

The FR group was treated with radiofrequency ablation of the maxillary nerve at the internal opening of the FR. The radiofrequency needle did not enter the Gasserian ganglion and the V1 and V3 branches were not affected, which was confirmed by the electrical stimulation test. Therefore, the $90^{\circ} \mathrm{C}$ temperature was selected for the radiofrequency ablation of the maxillary nerve. We had used this temperature in the hope of a more complete block of pain sensation conduction and better treatment efficacy. All 40 patients in the FR group showed no signs of postoperative injury of the V1 and V3 branches, and these patients had no facial pain in up to postoperative 1 year. These results suggest that higher temperature of radiofrequency ablation of the maxillary nerve is safe and effective for the treatment of V2 TN.

The FR group had significantly shorter operation time than the FO group $(19.3 \pm 5.9$ minutes vs. $32.7 \pm$ 8.7 minutes; $P<0.05)$. This is because radiofrequency ablation through the FO often requires multiple electrical stimulation tests to confirm that the V1 and V3 branches were not involved. The aim of electrical stimulation test in the FO group was to elicit paresthesia in the maxillary nerve dermatome, while minimizing it in the dermatomes of the V1 and V3 branches, thus reduc- ing the risks for corneal involvement and masticatory weakness. However, a radiofrequency needle inserted through the FR approach does not require multiple adjustment of the needle position, resulting in shorter operation time.

The FR group showed no signs of postoperative corneal involvement and masticatory weakness. However, 22 patients (55\%) and 31 patients $(78.5 \%)$ had corneal involvement and masticatory weakness postoperatively in the FO group. This suggests that radiofrequency ablation of the Gasserian ganglion through the FO involves significantly higher risks of neurologic complications than the FR approach. The incidence of facial numbness and swelling were comparable between the 2 groups. The symptoms of these 2 conditions were well tolerated and resolved spontaneously without special treatment.

Out study has limitations. First, the FR approach is difficult to learn. Second, radiofrequency thermocoagulation of the maxillary nerve through the FR is performed under fluoroscopy, which requires radiographic knowledge. Third, the nonrandomized design of our study may involve some bias.

\section{Conclusions}

In summary, radiofrequency thermocoagulation of the maxillary nerve through the FR under the guidance of DSA for the treatment of V2 TN had better efficacy and fewer complications in comparison with thermocoagulation of the Gasserian ganglion through the FO. The FR approach was also associated with significantly shorter operation time than the FO approach.

\section{Acknowledgments}

Author contributions: T.X., Q.Z., H.B., W.S., and C.Y. designed, performed this study, and wrote the article. P.Z., C.L., S.N., and Z.W. performed data collection and analyses. All authors read and approved the final manuscript. 


\section{References}

1. Nurmikko TJ. Toward an etiology-based management of trigeminal neuralgia. Pain Manag 2017; 7:149-154.

2. Yadav YR, Nishtha Y, Sonjjay P, Vigay P, Shailendra R, Yatin K. Trigeminal neuralgia. Asian J Neurosurg 2017; 12:585-597.

3. Cruccu G, Finnerup NB, Jensen TS, et al. Trigeminal neuralgia: New classification and diagnostic grading for practice and research. Neurology 2016; 87:220-228.

4. Du Y, Yu W. Comparison of nerve combing and percutaneous radiofrequency thermocoagulation in the treatment for idiopathic trigeminal neuralgia. Braz J Otorhinolaryngol 2017; 83:240.

5. Li X, Yue J, Yang L, et al. Application of antidromic conduction monitoring in ganglion radiofrequency thermocoagulation for locating trigeminal branches in trigeminal neuralgia. Pain Pract 2016; 16:305-310.

6. Fang L, Ying S, Tao W, Lan M, Xiaotong $Y$, Nan J. 3D CT-guided pulsed radiofre- quency treatment for trigeminal neuralgia. Pain Pract 2014; 14:16-21.

7. Dou Z, Zhang X, Yang L, et al. Alternation of regional homogeneity in trigeminal neuralgia after percutaneous radiofrequency thermocoagulation: A resting state fMRI study. Medicine (Baltimore) 2016; 95:e5193.

8. Huang B, Yao M, Feng Z, et al. CT-guided percutaneous infrazygomatic radiofrequency neurolysis through foramen rotundum to treat $V_{2}$ trigeminal neuralgia. Pain Med 2014; 15:1418-1428.

9. Wan Q, Zhang D, Cao X, Zhang Y, Zhu $M$, Zuo W. CT-guided selective percutaneous radiofrequency thermocoagulation via the foramen rotundum for isolated maxillary nerve idiopathic trigeminal neuralgia. J Neurosurg 2018; 128:211-214.

10. Chivukula S, Kim W, Zhuo X, et al. Radiosurgery for secondary trigeminal neuralgia: Revisiting the treatment paradigm.
World Neurosurg 2017; 99:288-294.

11. Ge H, Lv X, Jin H, He H, Li Y. Role of endovascular embolization for trigeminal neuralgia related to cerebral vascular malformation. Interv Neuroradiol 2016; 22:600-605.

12. Hayes DJ, Chen DQ, Zhong J, et al. Affective circuitry alterations in patients with trigeminal neuralgia. Front Neuroanat 2017; 11:73.

13. Tang $\mathrm{Y}, \mathrm{Ma} \mathrm{L}$, Li N, et al. Percutaneous trigeminal ganglion radiofrequency thermocoagulation alleviates anxiety and depression disorders in patients with classic trigeminal neuralgia: A cohort study. Medicine (Baltimore) 2016; 95:e5379.

14. Ding W, Chen S, Wang R, et al. Percutaneous radiofrequency thermocoagulation for trigeminal neuralgia using neuronavigation-guided puncture from a mandibular angle. Medicine (Baltimore) 2016; 95:e4940. 